\title{
LOCAL RIGIDITY OF SYMMETRIC SPACES
}

\author{
V. SCHROEDER AND W. ZILLER
}

\begin{abstract}
We show that on a symmetric space of noncompact or compact type the metric is locally rigid in the sense that if one changes the metric locally but preserves the curvature bounds, then the new metric is isometric to the old one. We also prove an analytic continuation property for symmetric spaces of rank $\geq 3$.
\end{abstract}

The aim of this paper is to show that a symmetric space is rigid under a local modification of the metric which preserves suitable curvature bounds. Our results were motivated by the following theorem of Gromov [BGS, §5]:

Theorem 1 (Gromov). Let $X$ be a simply connected symmetric space of rank $\geq 2$ and with nonpositive sectional curvature and $\Omega$ the complement of a compact set in $X$. If $f: \Omega \rightarrow Y$ is a totally geodesic isometric embedding, where $Y$ is a complete simply connected Riemannian manifold with nonpositive sectional curvature, then there exists a unique extension of $f$ to a totally geodesic isometric embedding $\bar{f}: X \rightarrow Y$.

We will give a slightly different proof of this theorem, which will then enable us to also prove the following dual result:

Theorem 2. Let $X$ be a simply connected symmetric space of rank $\geq 2$ and with sectional curvature $0 \leq K \leq 1$. If $Y$ is a complete manifold with curvature $K \geq 0$, and $\operatorname{dim} Y=\operatorname{dim} X$, and if $\Omega$ is the complement of a ball of radius $\pi / 30$ in $X$, then any isometric embedding $f: \Omega \rightarrow Y$ extends uniquely to an isometry $\bar{f}: X \rightarrow Y$.

If in the above two theorems, we choose $Y=X$ and $f=\mathrm{id}$, we obtain the desired local rigidity of symmetric spaces. Theorem 2 applies in particular to $S^{2} \times S^{2}$ and shows that the Hopf conjecture is at least locally correct.

The optimal radius for the ball in Theorem 2 would be the radius of a convex ball ( $\geq \pi / 2$ under those assumptions). Indeed, in the case of nonpositive curvature, Gromov [BGS, §5] proved the following local version of Theorem 1:

Received by the editors November 4, 1987 and, in revised form, September 12, 1988.

1980 Mathematics Subject Classification (1985 Revision). Primary 53C35; Secondary 53C20.

The first author is supported by the "Schweizerischer Nationalfond". The second author is partially supported by a grant from the National Science Foundation and would like to thank the IHES and the Max Planck Institut for its hospitality. 
Theorem 3 (Gromov). Let $X$ be a complete locally symmetric space of rank $\geq 2$ and with $K \leq 0$. Let $B$ be a compact simply connected convex region in $X$ and $U$ any neighborhood of $\partial B$. If $Y$ is complete with $K \leq 0$, then any totally geodesic isometric embedding $f: U \backslash B \rightarrow Y$ extends uniquely to a totally geodesic isometric embedding $\bar{f}: B \rightarrow Y$.

We conjecture, that the analogous result for $K \geq 0$ is also correct, but we can prove it only under the additional assumption that rank $\geq 3$ and $\partial B$ strictly convex:

Theorem 4. Let $X$ be a complete locally symmetric space of rank $\geq 3$ and with $K \geq 0$. Let $B$ be a compact, strictly convex region in $X$ and $U$ any neighborhood of $\partial B$. If $Y$ is complete with $K \geq 0$, then any isometric embedding $f: U \backslash B \rightarrow Y$ extends uniquely to an isometric embedding $\bar{f}: B \rightarrow Y$.

The method for proving Theorem 4 is a local version of the method for proving Theorem 2 (whereas the one in Theorem 3 is global). This local method also enables us to prove a certain analytic continuation property for locally symmetric spaces, which seems to be new also in the case of $K \leq 0$.

Theorem 5. Let $X$ be a complete manifold with $K \geq 0$ (resp. $K \leq 0), B$ a compact, simply connected, strictly convex region in $X$ and $U$ a neighborhood of $\partial B$. If the metric in $U \backslash B$ is locally symmetric of rank $\geq 3$, then the metric is also locally symmetric in $B$.

We emphasize that this result also holds locally in the following sense: If $H$ is a portion of a smooth strictly convex hypersurface, such that the metric on the concave side of $H$ is locally symmetric of rank $\geq 3$, then the metric on the convex side of $H$ is also locally symmetric, at least in a small neighborhood of $H$. Theorem 5 is an easy consequence of this local continuation property.

Theorem 5 is stronger than Theorems 3 and 4 (in the case where $Y=X$ and $f=\mathrm{id}$ ) since $X$ is not assumed to be locally symmetric. The complement of $B$ can be locally symmetric without being a portion of a complete locally symmetric space $X$. This is illustrated by the following well-known example, which also shows that Theorem 5 is in general false in the rank 2 case: There are complete metrics on $\mathbf{R}^{2}$ with $K \geq 0$ or $K \leq 0$ and flat outside a compact set, but not everywhere flat. A rounded off cone is such an example with $K \geq 0$ and one easily constructs such an example of the form $d r^{2}+f^{2}(r) d \theta^{2}$ with $K \leq 0$. But, if we assume that the complement of the compact set is isometric to a portion in $\mathbf{R}^{2}$ and not just flat, then the metric must be everywhere flat as follows from Theorem 1 and the proof of Theorem 2, or in dimension 2 just from the Gauss-Bonnet Theorem.

We do not know any higher dimensional counterexample to a rank 2 analogue of Theorem 5, although we will construct a metric on $\mathbf{R}^{n+1}$ with $K \geq 0$, which outside a compact convex region (not strictly convex) is locally isometric to the rank 2 symmetric space $\mathbf{R} \times S^{n}(1)$. The strict convexity of $\partial B$ in 
Theorem 5 is important, since a product of the above 2-dimensional counterexamples with a flat torus gives an example where $\partial B$ is only convex.

The following special consequence of Theorem 5 was proved earlier by Greene and Wu [GW] (see also Gromov [BGS, §4]): If $X$ is complete and simply connected with $K \leq 0, \operatorname{dim} X \geq 3$, and flat outside a compact set, then $X$ is isometric to $\mathbf{R}^{n}$. Notice that under these assumptions on $X$, every distance ball is strictly convex, and hence the result follows from Theorem 5 . In [BGS and GW] it is also shown that it is sufficient to assume that the curvature of $X$ goes to 0 faster than quadratically.

[GW] also show that if $K \geq 0, X$ is noncompact, flat outside a compact set, and simply connected at $\infty$, then $X$ is isometric to $\mathbf{R}^{n}$. If one drops the assumption that $X$ is simply connected at $\infty$, then one has the above twodimensional counterexamples or the product of such a two-dimensional example with a compact flat torus or a manifold covered by such an example. We will show that there are no other possibilities.

Theorem 6. Let $M$ be complete, noncompact with $K \geq 0$ and flat outside a compact set. Then either $M$ is flat or the universal cover splits isometrically as $X \times \mathbf{R}^{n-2}$, where $X$ is diffeomorphic to $\mathbf{R}^{2}$ with a metric with curvature $K \geq 0$ and flat outside a compact set. The fundamental group of $M$ is a Bieberbach group of rank $(n-2)$ and any soul of $M$ (in the sense of [CG]) is a compact, $(n-2)$-dimensional flat manifold.

In [BGS, p. 59] Gromov claims (without proof) that the conclusions of this theorem remain valid under the weaker condition that the curvature decays faster than quadratically.

For the general case of symmetric spaces, one can raise the question of classifying noncompact manifolds with $K \geq 0$ (or $K \leq 0$ ) such that $\|\nabla R\|$ decays sufficiently fast.

The case of noncompact, nonsimply connected manifolds with $K \leq 0$ which are flat outside a compact set are studied in detail in [ES]. There are more possibilities in this case than there are in Theorem 6.

The analytic continuation property in Theorem 5 is also valid for rank 1 symmetric spaces:

Theorem 7. Let $X$ be a complete manifold with $K \leq-1$ (resp. $K \geq 1$ ) and of dimension $\geq 3$. Let $B$ be a compact, simply connected, strictly convex region in $X$ and $U$ a neighborhood of $\partial B$. If the metric in $U \backslash B$ is locally symmetric of rank 1 , such that the maximum of the sectional curvature in $U$ is equal to -1 (resp. the minimum is equal to 1 ), then the metric in $B$ is locally symmetric of rank 1 .

This, of course, also implies again the local rigidity of rank 1 symmetric spaces as in Theorems 1 and 2 . In the case of $S^{n}(1)$, it says that one cannot change the metric within a hemisphere and still preserve $K \geq 1$. This result becomes false if $B$ is not convex, since one can glue a ball of radius $<\pi / 2$ in 
$S^{n}(1)$ with a ball of appropriate radius in $S^{n}(r), r<1$, to obtain a smooth manifold with $K \geq 1$ everywhere.

One also obtains an analytic continuation theorem for isometric embeddings of rank 1 symmetric spaces as in Theorems 3 and 4 .

If $X$ has dimension 2 , Theorem 7 is again false, but one can prove results analogous to Theorems 1 and 2: A theorem of Toponogov [T] implies that if $M^{2}$ has $K \geq 1$ and contains a set isometric to a hemisphere on $S^{2}(1)$, then $M^{2}$ is isometric to $S^{2}(1)$. We will show that if a metric on $\mathbf{H}^{2}$ has $K \leq-1$ and agrees with the standard metric on $\mathbf{H}^{2}$ outside a compact set, then $K \equiv-1$.

We would like to thank the referee for suggesting some improvements and Chris Croke and Herman Gluck for helpful discussions.

Proof of Theorems 1 and 2. We start with a proof of Theorem 1 on which the proof of the first five theorems are modelled.

Lemma 1. Let $\Omega$ be the complement of a compact set in $\mathbf{R}^{2}$ and $Y$ a complete, simply connected manifold with $K \leq 0$. Then any totally geodesic isometric embedding $f: \Omega \rightarrow Y$ extends uniquely to a totally geodesic isometric embedding $\bar{f}: \mathbf{R}^{2} \rightarrow Y$.

Proof. Let $\Delta$ be a triangle in $\mathbf{R}^{2}$ which surrounds the complement of $\Omega$. $f$ maps the edges of $\Delta$ to minimal geodesics in $Y$ and the angles of this triangle in $Y$ are the same as in the Euclidean case. Since $Y$ has nonpositive curvature the rigidity part of Rauchs comparison theorem implies that the geodesics in $Y$ are spanned by a totally geodesic, flat, embedded triangle isometric to $\Delta$ which agrees with $f(\Delta \cap \Omega)$ in $f(\Omega)$. Hence $f$ has a unique extension $\bar{f}: \mathbf{R}^{2} \rightarrow$ $Y$. Q.E.D.

If $X$ is a complete, simply connected manifold of nonpositive curvature, then we call an embedded, totally geodesic $\mathbf{R}^{2}$ in $X$ a 2-flat. We say that $X$ satisfies condition $(*)$ if for every tangent vector of $X$ there exists a 2-flat containing it and for any two 2-flats $F$ and $F^{*}$ through $x \in X$ there exists a sequence of 2-flats $F=F_{1}, F_{2}, \ldots, F_{k}=F^{*}$ such that $F_{i}$ and $F_{i+1}$ intersect along a geodesic through $x$.

Condition $(*)$ is satisfied for simply connected symmetric spaces of rank $\geq 2$ and $K \leq 0$ [BGS, Appendix 5 or the proof of Lemma 4b] and for nontrivial products $X_{1} \times X_{2}$ of simply connected manifolds with $K \leq 0$ [BGS, p. 69]. In [BGS] condition $(*)$ is formulated equivalently as follows: for any two geodesics $c, c^{*}$ through $x$, there exists a sequence of geodesics $c=$ $c_{1}, c_{2}, \ldots, c_{k}=c^{*}$ such that $c_{i}$ and $c_{i+1}$ are contained in a 2-flat.

Hence Theorem 1 is a consequence of the following:

Lemma 2. Let $X$ be a complete simply connected manifold with $K \leq 0$ and which satisfies (*). If $Y$ is a complete simply connected manifold with $K \leq$ 0 and $\Omega$ the complement of a compact set in $X$, then any totally geodesic isometric embedding $f: \Omega \rightarrow Y$ extends uniquely to a totally geodesic isometric embedding $\bar{f}: X \rightarrow Y$. 
Proof. If $x \in X \backslash \Omega$, let $F$ be a 2-flat through $x$. By Lemma $\left.1 f\right|_{F \cap \Omega}$ has a unique extension to $\tilde{f}: F \rightarrow Y$ and we define $\bar{f}(x)=\tilde{f}(x)$. To see that this definition is independent of the choice of $F$, we observe that if two 2flats intersect along a geodesic through $x$, this clearly gives the same value for $\tilde{f}(x)$ since the image of the geodesic in $f(\Omega)$ and hence in $Y$ is the same in both cases. Hence condition $(*)$ implies that $\bar{f}$ is well-defined. $\bar{f}$ is a totally geodesic isometric immersion since this is the case for $\tilde{f}: F \rightarrow Y$ and every vector is contained in a 2-flat. Q.E.D.

We now indicates the changes that are necessary to prove Theorem 2 .

It follows from [CE, 5.13] that a simply connected irreducible symmetric space with $0 \leq K \leq 1$ has injectivity radius $\geq \pi$ and hence the same is true for every simply connected symmetric space $X$ with $0 \leq K \leq 1$. A 2-flat in $X$ is now a totally geodesic isometric immersion $g: \mathbf{R}^{2} \rightarrow X$. Because of the duality for symmetric spaces, condition $(*)$ for 2 -flats holds in this case also. Hence it only remains to prove an extension analogous to Lemma 1.

Let $B=X \backslash \Omega$ the ball of radius $\pi / 30$ around $x_{0} \in X$. To define $\bar{f}$ at $y_{0} \in B$, choose a 2-flat $g: \mathbf{R}^{2} \rightarrow X$ with $g\left(y_{0}^{\prime}\right)=y_{0} \cdot g$ is an embedding on the ball $B_{\pi}\left(y_{0}^{\prime}\right)$ and we try to extend $\left.f\right|_{\Omega \cap g\left(B_{\pi}\left(y_{0}^{\prime}\right)\right)}$ to an isometric embedding $\tilde{f}: g\left(B_{\pi}\left(y_{0}^{\prime}\right)\right) \rightarrow Y$. For this purpose we surround $g\left(B_{\pi}\left(y_{0}^{\prime}\right)\right) \cap B$ by a triangle with minimal sides within $g\left(B_{\pi}\left(y_{0}^{\prime}\right)\right)$ : Choose an equilateral triangle $\Delta$ in $\mathbf{R}^{2}$ with center $y_{0}^{\prime}$ and such that the vertices of $\Delta$ have distance $\frac{17}{30} \pi$ from $y_{0}^{\prime}$. Hence $\Delta$ is contained within $B_{\pi}\left(y_{0}^{\prime}\right)$ and the sides of $\Delta$ have length $<\pi$. Thus $g(\Delta)$ is a triangle in $X$ with minimal sides. If $q$ is a vertex of $\Delta$, we have

$$
d\left(g(q), x_{0}\right) \geq d\left(g(q), y_{0}\right)-d\left(y_{0}, x_{0}\right) \geq \frac{17}{30} \pi-\frac{\pi}{30}
$$

and hence $d(g(q), B) \geq d\left(g(q), x_{0}\right)-\pi / 30 \geq \pi / 2$. The sides of $g(\Delta)$ are therefore contained in $\Omega$ and we claim that $f$ maps the sides of $\Delta$ into geodesics in $Y$ which are still minimal in $Y$. Indeed there are no shorter curves in $f(\Omega)$ since $f$ is an isometric embedding, and since we also have $d(f \circ g(q), Y-f(\Omega)) \geq \pi / 2$ for every vertex $q$ of $\Delta$, the sides are minimal in all of $Y$. Hence $f$ maps the sides of $g(\Delta)$ into a triangle $\Delta^{\prime}$. The rigid part of the Toponogov comparison theorem then implies that the sides of $\Delta^{\prime}$ are spanned by a totally geodesic, embedded, flat triangle isometric to $\Delta$, which in $f(\Omega)$ agrees with $f(g(\Delta) \cap \Omega)$. Hence $\left.f\right|_{\Omega \cap g\left(B_{\pi}\left(y_{0}^{\prime}\right)\right)}$ extends uniquely to $\tilde{f}: g\left(B_{\pi}\left(y_{0}^{\prime}\right)\right) \rightarrow Y$ and we define $\bar{f}\left(y_{0}\right)=\tilde{f}\left(y_{0}\right)$. That $\bar{f}$ is well-defined and a totally geodesic isometric embedding now follows as before from condition (*). Q.E.D.

Remarks. (1) As before, Theorem 2 is also true if $X$ is a nontrivial product $X_{1} \times X_{2}$ of complete manifolds with $K \geq 0$ and if the complement of $\Omega$ is a ball of radius $\operatorname{inj}\left(M_{1} \times M_{2}\right) / 30$. 
(2) In the case of $X=\mathbf{R}^{n}$, one can choose $\Omega$ to be the complement of an arbitrary compact set, since sufficiently large triangles in $g\left(\mathbf{R}^{2}\right) \cap \Omega$ will have minimal image in $Y$.

(3) For compact quotients of symmetric spaces with $K \geq 0$ one also has the Mostov rigidity theorem, which is unrelated to the above rigidity theorems. It states that on a compact quotient of an irreducible symmetric space with $K \leq 0$ and rank $\geq 2$, any metric with $K \leq 0$ must be symmetric. Such a theorem does not hold in the case of symmetric spaces with $K \geq 0$. Indeed, if $G$ is a compact simple Lie group of rank $\geq 2$, the metrics defined in [DZ, $\S 4]$ obtained from the bi-invariant metric on $G$ by multiplying it with a factor $t$ in the direction of a subgroup $K \subset G$ and its left translates, has sectional curvature $K \geq 0$ for $t \leq 1$, but is not isometric to the bi-invariant metric if $t<1$.

Proof of Theorems 4 and 5. The proof of Theorems 4 and 5 will be a local version of the proof of Theorems 1 and 2. We start with Theorem 4.

We first observe that there exists a family $B_{t}$ of compact strictly convex sets with $B_{0}=B$ and $B_{1}$ a point as follows from [CE, 8.9 and 8.10]. To construct the extension $\bar{f}: B \rightarrow Y$, it will be sufficient to show that for each $t$, if we have an extension of $f$ onto $B \backslash B_{t}$, then there exists a $t^{\prime}<t$ such that there exists an extension onto $B \backslash B_{t^{\prime}}$. For this it will be sufficient to show that for each $p \in \partial B_{t}$ there exists a $\sigma>0$ such that $f$ has an extension onto $B_{\sigma}(p)$. For simplicity we set $B=B_{t}$ again. To prove the extension onto $B_{\sigma}(p)$ we choose 2-flats near $p$ whose intersection with the interior of $B$ is very small, which is possible by the strict convexity of $B$. We then extend $f$ onto these 2-flats and show that there are sufficiently many such 2-flats to define the extension onto $B_{\sigma}(p)$. The condition rank $\geq 3$ is needed to guarantee the existence of sufficiently many 2-flats tangent to $\partial B$ at every $p \in \partial B$.

We start with the analogue of Lemma 1, which we formulate for arbitrary constant curvature, since this will be needed for the proof of Theorems 5 and 7 .

Lemma 3. Let $M^{2}(c)$ be a complete simply connected two-dimensional Riemannian manifold with constant curvature $c$ and $B_{r}(p)$ a ball of radius $r$ in $M^{2}$. Let $Y$ be a complete Riemannian manifold with sectional curvature $K$. If $c>0 \quad(c<0)$ we assume $K \geq c \quad(K \leq c)$ and if $c=0$ we assume either $K \geq 0$ or $K \leq 0$. If $A$ is a compact set in $Y$ let $i(A)$ be the injectivity radius of $A$. Then there exists an $\varepsilon>0$ depending on $r$ and $i(A)$ such that every totally geodesic isometric embedding $g: B_{r}(p) \backslash B_{\varepsilon}(p) \rightarrow A$ extends uniquely to a totally geodesic isometric embedding $\bar{g}: B_{r}(p) \rightarrow Y$.

Proof. Consider a geodesic triangle $\Delta$ in $B_{r}(p)$ such that the length of the sides are $\leq i(A)$ and such that $p$ is in its interior. Then there exists an $\varepsilon>0$ such that $B_{\varepsilon}(p)$ is in the interior of $\Delta$. By assumption $f$ maps the sides of $\Delta$ into minimal geodesics in $A \subset Y$ and hence $\Delta$ into a triangle $\widetilde{\Delta}$ in $Y$ whose angles are the same as the angles in $\Delta$. If $c>0$ or if $c=0$ and $K \geq 0$, we can use 
the rigid part of Toponogov to conclude that $\widetilde{\Delta}$ is spanned by an embedded totally geodesic surface isometric to the interior of $\Delta$ which then enables us to define the extension $\tilde{g}$ uniquely. If $c<0$ or $c=0$ and $K \leq 0$, we choose the triangle $\Delta$ such that the sides have length $\leq i(A) / 2$. Then $\widetilde{\Delta}$ is contained within a normal ball of $Y$ around one of its vertices and we can use the Rauch comparison theorem to conclude that $\widetilde{\Delta}$ is spanned by an embedded totally geodesic surface isometric to $\Delta$. Q.E.D.

Next we need something analogous to condition $(*)$ :

Lemma 4. Let $\bar{X}$ be a symmetric space of rank $\geq 3$ and $q \in \bar{X}$.

(a) For every $\delta \geq 0$, for every unit vector $N \in T_{q} \bar{X}$, and for every unit vector $v \in T_{q} \bar{X}$ with $\langle v, N\rangle \leq \delta$, there exists a 2-flat $F$ through $q$ with $v \in T_{q} F$ and $\langle w, N\rangle \leq \delta$ for all unit vectors $w \in T_{q} F$.

(b) Given a 2-flat $F$ through $q$, there exists a neighborhood $V$ of $F$ within the set of all 2-flats through $q$, such that for any $F^{\prime} \in V$ there exists a sequence of 2-flats $F_{1}, \ldots, F_{k} \in V$ with $F_{1}=F, F_{k}=F^{\prime}$ and $F_{i} \cap F_{i+1}$ is a geodesic through $q$ for each $i . V$ can be made arbitrarily small.

Proof. (a) Since the rank of $\bar{X}$ is $\geq 3$, there exists a 3-flat $Q$ containing $v \in T_{q} \bar{X}$. Let $w \in T_{q} Q$ be normal to $v$ and $N . v$ and $w$ are tangent to a 2-flat $F \subset Q$ which has the desired properties.

(b) It is sufficient to look at the tangent space of the 2-flats at $q$ and hence we only work in $T_{q} \bar{X}$. Let $\bar{X}=G / K, g=\ell+\mu$, and $a \subset \mu$ a maximal abelian subalgebra. $K$ acts $\mathrm{n} \mu$ and any maximal abelian subalgebra is equivalent to $a$ under an element of $K$. Hence, if $K_{a}$ is the normalizer of $a$ in $K$, then the set of maximal abelian subalgebras in $\rho$ is diffeomorphic to $K / K_{a}$. Let $\Delta$ be the set of nonzero roots of the symmetric space for the given maximal abelian subalgebra $a$, counted with multiplicity, and $\Delta_{+}$the set of positive roots in some ordering. Then there exist root vectors $X_{\alpha}^{+} \in R, Y_{\alpha} \in \rho$ with $h=h_{a}+\sum_{\alpha \in \Delta_{+}} \mathbf{R} \cdot X_{\alpha}, \mu=a+\sum_{\alpha \in \Delta_{+}} \mathbf{R} \cdot Y_{\alpha}$ and $\left[X_{\alpha}, A\right]=\alpha(A) Y_{\alpha}$ for $A \in a$. Hence $\operatorname{Ad}\left(\exp t X_{\alpha}\right) \in K$ rotates $a$ by leaving the singular hyperplane $\alpha=0$ in $a$ fixed, and rotating the vector perpendicular to it into $Y_{\alpha}$. Hence for any 2plane $F^{*} \subset a, F^{*}$ and $\operatorname{Ad}\left(\exp t X_{\alpha}\right) F^{*}$ have a vector in common. $\Delta_{+}$and $X_{\alpha}$ depend on the choice of $a$, but we can choose them, at least locally, such that $X_{\alpha}$ become smooth vector fields on $K / K_{a}$ near $a$. They then form a basis of the tangent space of $K / K_{a}$ at each point and we obtain maps $f\left(a^{\prime}, \alpha, t\right) \in K$ such that $F$ and $f\left(a^{\prime}, \alpha, t\right) F$ have a vector in common for each 2-plane $F \subset a^{\prime}$. It now easily follows that for each maximal abelian subalgebra $a^{\prime}$ near $a$, there exists $t_{1}, \ldots, t_{k}, k=\operatorname{dim} K / K_{a}$, with $a_{1}=a, a_{i+1}=f\left(a_{i}, \alpha_{i}, t_{i}\right) a_{i}$, and $a_{k+1}=a^{\prime}$ and hence $a^{\prime}=\prod_{i=1}^{k} f\left(a_{i}, \alpha_{i}, t_{i}\right) a$. Now let $F$ be the given 2-flat and choose $a$ with $F \subset a$. Let $W(F)$ be a neighborhood of $F$ within the set of 2-planes in $a$. We can choose $W$ such that for any $Q \in W$, there exists a $F^{*} \in W$ with $\operatorname{dim} F \cap F^{*}=\operatorname{dim} F^{*} \cap Q=1$. Given a small $\varepsilon>0$, consider the set $V$ of 2-planes of the form $\prod_{i=1}^{k} f\left(a_{i}, \alpha_{i}, t_{i}\right) Q$ with $Q \in W(F),\left|t_{i}\right| \leq \varepsilon$, 
and $a_{i+1}=f\left(a_{i}, \alpha_{i}, t_{i}\right) a_{i}, a_{1}=a$. Then $V$ is a neighborhood of $F$ within the set of 2-flats in $\rho$ since any $F^{\prime}$ near $F$ is contained in an $a^{\prime}$ near $a$. By construction, the set $V$ satisfies the properties in Lemma 4(b). By choosing $\varepsilon$ and $W(F)$ small enough, $V$ can be made as small as desired. Q.E.D.

Remark. The proof of Lemma 4(b) does not use rank $\geq 3$ and hence also gives a proof of the previous condition * since the set of all 2-flats through $q$ is connected.

We now start with the proof of Theorem 4. As explained earlier, it is sufficient to show that for each $p \in \partial B$, there exists an extension of $f$ onto $B_{\sigma}(p)$ for some $\sigma>0$. Let $N$ be the outside unit normal to $\partial B$ at $p$, or, if $\partial B$ is not smooth at $p$, a unit normal to any supporting hyperplane of $B$ at $p$. We can define 2-flats in $X$ at least locally: A neighborhood of $p$ is isometric to a neighborhood in a globally symmetric space $\bar{X}$ and a 2-flat in $X$ near $p$ is defined to be the image of a connected portion of a 2-flat in $\bar{X}$.

By Lemma 4(a), for each $\delta>0$ we can choose a compact set $S$ of 2-flats in $X$ through $p$ such that for each unit vector $v \in T_{p} X$ with $\langle v, N\rangle \leq \delta$ there exists a 2-flat in $S$ containing $v$ and such that for every 2-flat $F \in S$ and for every unit vector $w \in T_{p} F$ we have $\langle w, N\rangle \leq \delta$. One easily shows that this set $S$ can also be chosen to be connected. By Lemma 4(b), we can choose a neighborhood $V$ of $S$ within the set of all 2-flats through $p$ such that $F$, $F^{*} \in V$ are connected by a sequence of 2-flats in $V$ with two consecutive ones intersecting along a geodesic through $p$.

If $G$ is the set of isometries of $\bar{X}$, then a neighborhood $W$ of $e \in G$ acts as local isometries near $p \in X$ and for $g \in W$ we will use the 2-flats $g \cdot F$, $F \in V$, near $g \cdot p$.

We now consider the neighborhood $U$ of $\partial B$, assume that the isometric embedding $f: U \backslash B \rightarrow Y$ is defined and try to extend it to $B_{\sigma}(p) \cap U$. By choosing $W$ sufficiently small, we can find $r>0$ such that every 2-flat $g$. $F, g \in W, F \in V$ is defined on a ball of radius at least $r$ and such that $B_{r}(g \cdot p) \cap g \cdot F \subset U$. We can assume, by the strict convexity of $\partial B$ and by making $r$ smaller if necessary, that $B_{r}(p) \cap F$ intersects $\partial B$ only at $p$ for any 2-flat $F$ which is perpendicular to $N$. Since any $F \in S$ is almost normal to $N, B_{\sigma}(p) \cap F$ interests $B$ only in a small set and the same is true for any $F \in V$ if we choose $V$ sufficiently small. Hence we can choose $W, \delta$, and $V$ sufficiently small such that $B_{r}(g \cdot p) \cap g \cdot F \cap B$ is contained in $B_{\varepsilon}(g \cdot p)$ for any $g \in W, F \in V$, where $\varepsilon$ is the constant in Lemma 3. Then the restriction of $f$ to $\left(B_{r}(g p) \backslash B_{\varepsilon}(g p)\right) \cap g F$ extends uniquely to a totally geodesic isometric embedding $f^{*}: B_{r}(g p) \cap g F \rightarrow Y$ by Lemma 3 .

If $g, g^{\prime} \in W$ with $g p=g^{\prime} p$, then $g=g^{\prime} \cdot k$ for some $k \in K$. We choose $V^{\prime} \subset V$ and $W$ sufficiently small such that $S \subset V^{\prime}$ and for any $g, g^{\prime} \in W$ and $F \in V^{\prime}$ we have $\left(\left(g^{\prime}\right)^{-1} \cdot g\right) \cdot F \in V$. Now let $\sigma>0$ be such that $B_{\sigma}(p) \subset\{g p \mid g \in W\}$. For $q \in B_{\sigma}(p)$, choose $g \in W$ with $g p=q$ and for any $F \in V^{\prime}$ define $\bar{f}(q)=f^{*}(q)$ where $f^{*}: B_{r}(g p) \cap g F \rightarrow Y$ is the 
above extension. $\bar{f}(q)$ is independent of the choice of $F \in V^{\prime}$, since for $F$, $F^{*} \in V^{\prime}, g \cdot F$ and $g \cdot F^{*}$ can be connected by a sequence of 2-flats $g \cdot F^{\prime}$, $F^{\prime} \in V$, with two consecutive ones intersecting along a geodesic through $g p$. $\bar{f}(q)$ is also independent of the choice of $g$, since $g p=g^{\prime} p$ implies $g=g^{\prime} k$ and hence $g F=g^{\prime} k F=g^{\prime} F^{*}$ with $F^{*} \in V$ by assumption, and as before $g^{\prime} F$ and $g^{\prime} F^{*}$ give rise to the same extension at $q$.

To show that $\bar{f}$ is also a totally geodesic isometric embedding we first observe that $\bar{f}$ and $f^{*}$ agree on all of $B_{r}(g p) \cap g F$. Indeed, if $q \in B_{r}(g p) \cap g F$ then the transvection along the geodesic from $q$ to $p$ is an isometry that takes the tangent space of the 2-flat at $q$ to the tangent space of the 2-flat at $g p$, since the derivative of a transvection is given by parallel translation along the geodesic. Hence we can assume that there exists a $g^{\prime} \in W$ with $g^{\prime} p=q$ and $g^{\prime} F$ agrees with $g F$ near $q$ which shows that $\frac{g}{f}(q)=f^{*}(q)$. Hence $\frac{q}{f}$ preserves the length of every vector tangent to $g F$ at $g p$. Next observe that $\bar{f}$ is differentiable. Indeed, we can describe each $q \in B_{\sigma}(p)$ in a differentiable fashion as the midpoint of a geodesic $\gamma:[0,1] \rightarrow X$ with $\gamma(0), \gamma(1) \in U \backslash B$ and $\bar{f}\left(\gamma\left(\frac{1}{2}\right)\right)$ is then the midpoint of the unique geodesic connecting $f(\gamma(0))$ and $f(\gamma(1))$. We now want to show that $L=d \bar{f}_{q}$ is a linear isometry. For every unit vector $y \in T_{p} X$ with $\langle N, y\rangle \leq \delta$ there exists a 2-flat $F \in S \subset$ $V^{\prime}$ containing $y$. Hence $\|L y\|=\|y\|$ for every unit vector $y \in T_{q} X$ with $\langle g N, y\rangle<\delta$. If we choose a basis $e_{i}$ of $T_{q} X$ with $\left\langle g N, e_{i}\right\rangle<\delta / 2$, then $\left\|L e_{i}\right\|=\left\|e_{i}\right\|$ and $\left\|L\left(e_{i}+e_{j}\right)\right\|=\left\|e_{i}+e_{j}\right\|$ and hence $\left\langle L e_{i}, L e_{j}\right\rangle=\left\langle e_{i}, e_{j}\right\rangle$ or $L$ is an isometry. Similarly one shows that $\bar{f}$ is totally geodesic. This finishes the proof of Theorem 4 .

We now indicate the changes needed to prove Theorem 5 . As before it suffices to show that if the metric is locally symmetric in $U \backslash B$, then for each $p \in \partial B$, the metric is locally symmetric in $B_{\sigma}(p)$ for some $\sigma>0$. (In the case of $K \leq 0$, the existence of the family $B_{t}$ with $B_{1}=$ point follows since $B$ lifts isometrically to the universal cover $\tilde{X}$ because $B$ is simply connected. In $\widetilde{X}$ all balls are strictly convex and hence a family of balls whose radius shrinks to 0 intersected with $B$ will be such a family in $X$.)

Let $N$ be an outside unit normal of $\partial B$ at $p$ and $\gamma(t)=\exp t N$. Let $\varepsilon>0$ be such that $B_{2 \varepsilon}(\gamma(\varepsilon)) \subset U$. If $\varepsilon$ is sufficiently small, then every minimal geodesic from $\gamma(\varepsilon)$ to $\partial B \cap B_{2 \varepsilon}(\gamma(\varepsilon))$ does not intersect the interior of $B$. In a neighborhood of $\gamma(\varepsilon), X$ is isometric to a neighborhood in a globally symmetric space $\bar{X}$. This isometry can be extended along every geodesic in $U \backslash B$ starting at $\gamma(\varepsilon)$. Hence there exists an isometry $g: B_{2 \varepsilon}(\gamma(\varepsilon)) \backslash B \rightarrow \bar{X}$ whose image is of the form $B_{2 \varepsilon}(q) \backslash \bar{B}$ where $\bar{B}$ has strictly convex boundary in $B_{2 \varepsilon}(q) \subset \bar{X}$. Now we can apply the same methods as in the proof of Theorem 4, to extend the isometry $g^{-1}$ to a neighborhood of $g(p)$ and hence $X$ is locally symmetric in a neighborhood of $p$.

Remark. All rank 2 symmetric spaces, except for the product of a rank 1 symmetric space with a real line, still have the property that orthogonal to every 
vector there is a 2-flat. This follows, e.g., from Kostant's convexity theorem [K]. But, to show that the extension is an isometry, we need the stronger condition in Lemma 4(a) which is never satisfied for a rank 2 symmetric space.

Example. We now indicate the example on $\mathbf{R}^{n+1}$ with $K \geq 0$ which is locally isometric to $\mathbf{R} \times S^{n}$ outside a compact set.

It is a warped product metric on $\mathbf{R}^{+} \times S^{n}$ of the form $d t^{2}+f^{2}(t) g$ where $g$ is a metric on $S^{n}$ with $K \equiv 1$. If $X$ is a vector tangent to $\mathbf{R}$ and $U, V$ vectors tangent to $S^{n}$, one easily shows that the sectional curvatures are given by $K(X, U)=-f^{\prime \prime} / f$ and $K(U, V)=\left(1-f^{\prime 2}\right) / f^{2}$. See, e.g. [B, p. 265]. If only one of the four vectors in the full curvature tensor is tangent to $\mathbf{R}$, then it vanishes and hence $K \geq 0$ if $f^{\prime \prime} \leq 0$ and $f^{\prime} \leq 1$. We can make the metric near $t=0$ smooth (and flat) by letting $f(t)=t$ for $t$ small. For $t$ large we can make $f(t)=$ constant $=1$ and still preserve $f^{\prime \prime} \leq 0$ and $f^{\prime} \leq 1$ for all $t$. Notice that the balls $t \leq a$ are convex for any $a$ but not strictly convex for any $a$ with $f(a)=1$.

Remark. Theorem 3 follows as in Theorem 1 from the following extension lemma [BGS]: Let $B$ be a compact, simply connected, convex region in $\mathbf{R}^{2}, U$ a neighborhood of $\partial B$ and $Y$ a complete manifold with $K \leq 0$. Then every totally geodesic isometric embedding $f: U \rightarrow Y$ extends uniquely to a totally geodesic isometric embedding $\bar{f}: B \rightarrow Y$. It is conceivable that this extension lemma is still correct for $K \geq 0$, which would prove the analogue of Theorem 3 for $K \geq 0$. It would also be interesting to know if the corresponding extension lemmas for $K \geq 1$ and $K \leq-1$ are valid.

Proof of Theorem 7. The proof of Theorem 7 is similar to the one of Theorem 5. For a rank 1 symmetric space with $\max K=-1(\operatorname{resp} . \min K=1)$ the 2flats are replaced by totally real 2-planes, i.e., totally geodesic immersed 2dimensional submanifolds of curvature -1 (resp. 1). In the noncompact case they are embedded and isometric to $\mathbf{H}^{2}$, in the compact case they are isometric to an embedded $S^{2}(1)$ if $X=S^{n}$ and isometric to an embedded $\mathbf{R} P^{2}$ for the projective spaces. We claim that there are again sufficiently many of these totally real 2-planes in order to prove the analogue of Lemma 4: By the duality for symmetric spaces it is sufficient to do this for the hyperbolic spaces $\mathbf{H}_{\mathbf{K}}^{n}, \mathbf{K}=\mathbf{R}$, $\mathbf{C}, \mathbf{H}, \mathbf{Q}$. If $\mathbf{K}=\mathbf{R}$, Lemma 4 is obvious. In the other cases we can hence assume $n>1$. Let $v$ be a tangent vector at $q$. Then $v$ is tangent to a 2,4 , or 8-dimensional totally geodesic submanifold of constant curvature -4 . We denote the tangent space of this submanifold at $q$ by $\mathbf{K} v$ and let $\mathbf{K}^{\perp} v$ be the hyperplane in $\mathbf{K} v$ perpendicular to $v, v$ and $w$ span a totally real subspace (and hence are tangent to a totally real 2-plane) iff $w$ is orthogonal to $\mathbf{K}^{\perp} v$. Now let $N, v$ be given with $\langle N, v\rangle=0$ and choose $w$ perpendicular to $\mathbf{K}^{\perp} v$ and $N$, but not proportional to $v$, which is possible for dimension reasons. Then $v$ and $w$ span a totally real subspace orthogonal to $N$ and containing $v$. This proves Lemma $4(\mathrm{a})$. 
To prove Lemma $4(\mathrm{~b})$, we first assume $\mathbf{K}=\mathbf{C}$ or $\mathbf{H}$. If we identify $T_{q} X$ with $\mathbf{K}^{n}$, then $\mathbf{K}^{\perp} v$ is spanned by $i \cdot v$ if $\mathbf{K}=\mathbf{C}$, and by $i \cdot v, j \cdot v, k \cdot v$ if $\mathbf{K}=\mathbf{H}$. (This uses the associativity of $\mathbf{K}$ and is false for $\mathbf{K}=\mathbf{Q}$.) Let now $F$ be a totally real 2-plane in $\mathbf{K}^{n}$ and $e_{1}, e_{2}$ an orthonormal basis of $F$. Then $\left\langle\mathbf{K} e_{1}, e_{2}\right\rangle=0$ and hence $\left\langle e_{1}, \mathbf{K} e_{2}\right\rangle=0$. Every 2-plane $L$ near $F$ can be uniquely written as the graph of a linear map $A$ from $F$ to $F^{\perp}$, i.e. $e_{1}+A\left(e_{1}\right), e_{2}+A\left(e_{2}\right)$ is a basis of $L$. Since $K^{\perp} e_{1}$ and $K^{\perp} e_{2}$ are contained in $F^{\perp}$ and are perpendicular to each other, we can write $A\left(e_{i}\right)=a_{i} e_{1}+b_{i} e_{2}+u_{i}$ where $a_{i}, b_{i} \in \mathbf{K}^{\perp}=\operatorname{Im}(\mathbf{K})$ and $u_{i}$ is perpendicular to the span of $\mathbf{K} e_{1}$ and $\mathbf{K} e_{2}$.

We first observe that for any $a, b \in \mathbf{K}^{\perp}$ and $u \in\left(\mathbf{K} e_{1}+\mathbf{K} e_{2}\right)^{\perp}$, the 2-plane spanned by $e_{1}+a e_{1}+b e_{2}+u$ and $e_{2}+b e_{1}+a e_{2}$ is totally real. Indeed, a calculation shows that $k \cdot\left(e_{2}+b e_{1}+a e_{2}\right)$ is perpendicular to $e_{1}+a e_{1}+b e_{2}+u$ for any $k \in \mathbf{K}^{\perp}$ :

$$
\begin{aligned}
\left\langlek \left( e_{2}\right.\right. & \left.\left.+b e_{1}+a e_{2}\right), e_{1}+a e_{1}+b e_{2}+u\right\rangle \\
& =\left\langle k e_{2}, b e_{2}\right\rangle+\left\langle k b e_{1}, e_{1}\right\rangle+\left\langle k b e_{2}, a e_{1}\right\rangle+\left\langle k a e_{2}, b e_{2}\right\rangle \\
& =\langle k, b\rangle+\langle k b, 1\rangle+\langle k b, a\rangle+\langle k a, b\rangle=0 .
\end{aligned}
$$

Here we have used the fact that $\langle a e, b e\rangle=\langle a, b\rangle$ if $a, b \in \mathbf{K}, e \in \mathbf{K}$ with $|e|=1$ and that $\langle a b, c\rangle=\langle b, \bar{a} c\rangle$ if $a, b, c \in \mathbf{K}$.

Hence we define 2-planes $F_{1}, F_{2}, F_{3}$ where $F_{1}$ is spanned by $e_{1}$ $+\frac{1}{2}\left(a_{1}+b_{1}\right) e_{1}+\frac{1}{2}\left(a_{1}+b_{1}\right) e_{2}$ and $e_{2}+\frac{1}{2}\left(a_{1}+b_{1}\right) e_{1}+\frac{1}{2}\left(a_{1}+b_{1}\right) e_{2}, F_{2}$ is spanned by $e_{1}+a_{1} e_{1}+b_{1} e_{2}$ and $e_{2}+b_{1} e_{1}+a_{1} e_{2}$, and $F_{3}$ is spanned by $e_{1}+a_{1} e_{1}+b_{1} e_{2}+u_{1}$ and $e_{2}+b_{1} e_{1}+a_{1} e_{2}$. The above comment shows that $F_{1}, F_{2}, F_{3}$ are totally real. $F$ and $F_{1}$ have $e_{1}-e_{2}$ in common, $F_{1}$ and $F_{2}$ have $\left(1+a_{1}+b_{1}\right) e_{1}+\left(1+a_{1}+b_{1}\right) e_{2}$ in common, $F_{2}$ and $F_{3}$ have $e_{2}+b_{1} e_{1}+a_{1} e_{2}$ in common, and $F_{3}$ and $L$ have $e_{1}+a_{1} e_{1}+b_{1} e_{2}+u_{1}$ in common. Hence we have connected $F$ to $L . L$ is near $F$ iff all entries of $A$ are small. But then $F_{1}, F_{2}$, and $F_{3}$ are also near $F$ by construction. This proves Lemma $4(\mathrm{~b})$ in the case of $\mathbf{K}=\mathbf{C}$ or $\mathbf{H}$.

If $\mathbf{K}=\mathbf{Q}$ and $n=2$, the above calculation needs to be modified. We still describe $T_{q} X$ as $\mathbf{Q}^{2}$, but now $\mathbf{K} v$ is of the form $H^{-1}(H(v))$, where $H$ is the Hopf map: $\mathbf{Q}^{2} \rightarrow \mathbf{Q} \cup \infty, H(a, b)=a^{-1} b$ if $a \neq 0$ and $H(0, b)=\infty$. Hence $\mathbf{K}(a, b)=\left\{\left(v, v\left(a^{-1} b\right)\right) \mid v \in \mathbf{Q}\right\}$ and one easily checks that $\mathbf{K}^{\perp}(a, b)=$ $\left\{\left(v, v\left(a^{-1} b\right)\right) \mid v \in \mathbf{Q},\langle v, a\rangle=0\right\}$. For a description of the Hopf fibration and some of its properties, as well as some rules for computing with $\mathbf{Q}$ we refer to [GWZ]. To simplify the calculation, we first bring $F$ into standard form. The group of symmetries of the Hopf fibration, i.e. the group of linear isometries preserving the fibres of $H$, clearly takes totally real 2-planes to totally real 2planes. This group is equal to $\operatorname{Spin}(9)$, see [GWZ], and one easily shows that it acts transitively on the set of totally real 2-planes. Hence we can assume that $F$ is spanned by $e_{1}=(1,0)$ and $e_{2}=(0,1)$. A 2-plane $L$ near $F$ is 
described as the graph of a linear map $A$ from $F$ to $F^{\perp}: A\left(e_{i}\right)=\left(a_{i}, b_{i}\right)$, $a_{i}, b_{i} \in \mathbf{Q}^{\perp}$. In analogy with the previous case, we claim that $L$ is totally real if $b_{2}=a_{1}$ and $a_{2}=b_{1}$ or in other words, we claim that the 2-plane spanned by $(b, 1+a)$ and $(1+a, b)$ is totally real for any $a, b \in \mathbf{Q}^{\perp}$. To prove this we have to show that

$$
\left\langle\mathbf{Q}^{\perp}(b, 1+a),(1+a, b)\right\rangle=0 \quad \text { or } \quad\left\langle\left(v, v\left[b^{-1}(1+a)\right]\right),(1+a, b)\right\rangle=0
$$

for all $v \in \mathbf{Q}$ with $\langle v, b\rangle=0$. But

$$
\begin{aligned}
\langle(v, v & {\left.\left.\left[b^{-1}(1+a)\right]\right),(1+a, b)\right\rangle } \\
& =\langle v, 1+a\rangle+\left\langle v\left[b^{-1}(1+a)\right], b\right\rangle \\
& =\langle v, 1+a\rangle+\left\langle b^{-1}(1+a), \bar{v} b\right\rangle \\
& =\langle v, 1+a\rangle-\left\langle b^{-1}(1+a), \bar{b} v\right\rangle \\
& =\langle v, 1+a\rangle-\langle 1+a, v\rangle=0 .
\end{aligned}
$$

Here we used the fact that $\langle a b, c\rangle=\langle b, \bar{a} c\rangle$ still holds for $\mathbf{Q}$ and that $\langle a, b\rangle=$ 0 implies $\bar{a} b=-b \bar{a}$. We can now proceed as before, by defining $F_{1}, F_{2}, F_{3}$. This finishes the proof of Lemma 4 (b) for $\mathbf{K}=\mathbf{Q}$.

The rest of the proof of Theorem 7 is now as before. Q.E.D.

Remark. There also exists a partial local rigidity for the lower curvature bound of $\mathbf{H}^{n}$ and the upper curvature bound of $S^{n}$ :

Let $\Omega$ be the complement of a sufficiently small compact set in $\mathbf{H}^{n}$ and let $Y$ be a complete simply connected manifold with $-1 \leq K \leq 0$. Then every totally geodesic isometric embedding $f: \Omega \rightarrow Y$ extends to a totally geodesic isometric embedding $\bar{f}: \mathbf{H}^{n} \rightarrow Y$. The complement of $\Omega$ must be small enough such that its intersection with every totally geodesic $\mathbf{H}^{2}$ in $\mathbf{H}^{n}$ lies in the interior of some geodesic triangle.

Similarly, if $\Omega$ is the complement of a ball of radius $r<\pi / 4$ in $S^{n}$ and $Y$ a complete simply connected $n$-dimensional manifold with $1 / 4 \leq K \leq 1$ or $0<K \leq 1$ and $n$ even, then every isometric embedding $f: \Omega \rightarrow Y$ extends to an isometry $\bar{f}: S^{n} \rightarrow Y$. Without such additional lower curvature bounds, this becomes false since one easily constructs surfaces of revolution with $K \leq 1$ and containing an arbitrarily large portion of $S^{2}$. We do not know if such examples also exist in higher dimension.

The proof of both statements is similar to the proof of Theorems 1 and 2 .

Dimension 2. We finally make some comments about the 2-dimensional case.

We already remarked that there exist metrics on $\mathbf{R}^{2}$ which are flat outside a compact set with $K \geq 0$ or $K \leq 0$ but which are not flat. But if we make the stronger assumption that outside the compact set the metric is globally isometric to the complement of a compact set in $\mathbf{R}^{2}$, then the metric must be everywhere flat. This follows from Theorem 1 and the proof of Theorem 2, or even simpler directly from Gauss and Bonnet. The situation is similar for $S^{2}$ and $\mathbf{H}^{2}$ : 
A theorem of Toponogov states [T] that a metric on $M^{2}$ with $K \geq 1$ which has a closed geodesic of length $2 \pi$ and without self-intersections must be isometric to a round sphere. This implies that if we change the metric on $S^{2}$ within a strictly convex set and preserve $K \geq 1$, then the new metric is isometric to the old one. Although this result is also true in higher dimensions by Theorem 5, Toponogov's proof does not carry over to higher dimensions and our proof does not work in dimension 2. Of course, Theorem 7 is also stronger since we only need to assume that $K=1$ outside the strictly convex set. Theorem 7 is false in dimension 2 since one can round off a surface of revolution with $K=1$ at their singularities such that $K \geq 1$.

We claim that there also exists such a theorem for $\mathbf{H}^{2}$ : If a metric on $\mathbf{H}^{2}$ has $K \leq-1$ and agrees with the standard metric outside a compact set $A$, then $K \equiv-1$ everywhere. We only indicate a proof. Since $A$ is arbitrarily large, we may not be able to surround $A$ by a geodesic triangle, but we can surround $A$ by a geodesic polygon. We claim that there exists a geodesic polygon $P$, which we can make as large as necessary, such that any geodesic polygon $P^{*}$ whose sides have the same length as the corresponding sides in $P$ and whose angles are greater or equal to the corresponding angles in $P$, must be isometric to $P$. Once we have such a $P$, we surround $A$ with $P$, subdivide $P$ into triangles and construct comparison triangles in $\mathbf{H}^{2}$ (with $K \equiv-1$ ) which then form a polygon $P^{*}$ whose sides have lengths the same as in $P$ and whose angles are greater or equal to the ones in $P$. Hence $P^{*}$ is isometric to $P$ and hence $K \equiv-1$ inside $P$.

Although it does not seem to be true that every convex polygon $P$ has this property ( $P^{*}$ does not need to be convex!) one can show that for every $n$ the convex polygon in $\mathbf{H}^{2}$ given by vertices $q_{0}, q_{1}, \ldots, q_{n}$ with $d\left(q_{0}, q_{i}\right)=n$ for $i=1, \ldots, n$ and $d\left(q_{i}, q_{i+1}\right)=1$ for $i=1, \ldots, n-1$ has this property. The proof we have is a somewhat tedious calculation using induction on $n$, and we leave it as an exercise to the reader.

Proof of Theorem 6. Let $M^{n}$ be a complete noncompact Riemannian manifold with $K \geq 0$ and $K \equiv 0$ outside a compact set and assume that $M$ is not flat everywhere. [CG, Theorem 6.2] states that if $K \geq 0$ then one can decompose the universal cover $\widetilde{M}$ isometrically as $X \times \mathbf{R}^{k}$, such that the isometry group of $X$ is compact. Since $M$ is noncompact and flat outside a compact set, $X$ is noncompact also. Let $\pi: \widetilde{M} \rightarrow M$ be the canonical projection. Since the isometry group of $X$ is compact, $\pi: X \times\{0\} \rightarrow M$ is proper, and hence the metric on $X$ is flat outside a compact set. Let $S$ be a soul of $X$ in the sense of [CG]. We will show that $\operatorname{dim} X=2$ and that $S$ is a point.

First we claim that the codimension of $S$ is equal to 2 . Indeed, if the codimension is 1 , Theorem 4.2 in [CG] implies that $X$ is isometric to the normal bundle of $S$ with its natural flat bundle metric. But since $X$ is flat outside a compact set, this would imply that $S$ and hence $X$ is flat. Now assume that $\operatorname{codim} S \geq 3$. Since $X$ is simply connected, and diffeomorphic to the normal 
bundle of $S$ by [CG], $S$ is also simply connected and hence $\operatorname{codim} S \geq 3 \mathrm{im}$ plies that $X$ is simply connected at infinity. Choose a compact set $K \subset X$ such that $X \backslash K$ is simply connected and flat. Then one gets an isometric immersion $f: X \backslash K \rightarrow \mathbf{R}^{n}$. Since $f(\partial(X \backslash K))$ is compact, we can choose a compact set $K^{\prime} \subset \mathbf{R}^{n}$ such that $f(\partial(X \backslash K)) \subset K^{\prime}$ and $\mathbf{R}^{n} \backslash K^{\prime}$ is simply connected. One checks easily that $f^{-1}\left(\mathbf{R}^{n} \backslash K^{\prime}\right)=X \backslash \bar{K}$ for some compact set $\bar{K} \supset K$ and that $f: X \backslash \bar{K} \rightarrow \mathbf{R}^{n} \backslash K^{\prime}$ is a covering map. Since $\mathbf{R}^{n} \backslash K^{\prime}$ is simply connected, $f: M \backslash \bar{K} \rightarrow \mathbf{R}^{n} \backslash K^{\prime}$ is a global isometry and Remark (2) after Theorem 1 applied to $f^{-1}$ shows that $f^{-1}$ can be extended to an isometry of $\mathbf{R}^{n}$ onto $M$.

Hence we can assume $\operatorname{codim} S=2$, and we want to show that $S$ consists of a point. We first make the following general comments.

Let $h:[0, \infty) \rightarrow X$ be a ray parametrized by arc length with $h(0) \in S$. Then [CE, 8.22(3)] implies that $h^{\prime}(0)$ is perpendicular to $S$. Furthermore let $c: \mathbf{R} \rightarrow S$ be a geodesic in $S$ with $c(0)=h(0)$ and $V(t)$ the parallel vector field along $c$ with $V(0)=h^{\prime}(0)$. Then [CE, 8.22(4)] implies that $\phi: \mathbf{R} \times[0, \infty) \rightarrow$ $X, \phi(t, s)=\exp _{c(t)} s \cdot V(t)$, is a totally geodesic isometric immersion and the geodesics $h_{t}(s)=\phi(t, s)$ are rays for all $s$. Let $p \in S$ and $h$ a ray with $h(0)=p$ and hence $h^{\prime}(0)=v$ perpendicular to $S$. We look at the orbit of $v$ under parallel translation along piecewise geodesics in the soul. If $w$ is in its orbit, then by the above remark, the geodesic $s \rightarrow \exp w$ is a ray for $s \geq 0$. Since $\operatorname{codim} S=2$ and $S$ is simply connected there are only two possibilities:

(A) The orbit of $v$ is a globally defined normal parallel vector field along $S$.

(B) The orbit of $v$ is the whole unit normal bundle $N_{1}(S)$ of $S$ in $X$.

In case (A) we obtain a totally geodesic isometric immersion $\psi: S \times[0, \infty) \rightarrow$ $X$ and hence we have totally geodesic copies $S \times\{t\}$ of $S$ in $X$. Since $X$ is flat outside a compact set, $S$ is also flat. Since $S$ is simply connected and compact, $S$ must be a point. It remains to consider case (B). In this case it follows that all geodesics normal to $S$ are rays and hence the normal exponential map is a diffeomorphism of $N_{1}(S)$ onto $X$. Thus $H_{t}=\{x \in X \mid d(x, s)=t\}$ is a smooth embedded submanifold of $X$ for all $t>0$. Choose $t$ large enough such that $H_{t}$ lies in the set where $X$ is flat. Let $W$ be a local unit normal vector field along $H_{t}$. We claim that $W$ is parallel in $X$ and hence $H_{t}$ is totally geodesic. Let $p \in H_{t}$ and choose a normal neighborhood $U$ of $p$ such that $K \equiv 0$ on $U$. Let $W^{*}$ be the parallel vector field on $U$ with $W^{*}(p)=W(p)$. We want to show that $W=W^{*}$ on $U \cap H_{t}$. First we claim that for any $q \in U \cap H_{t}$, there exists a piecewise geodesic $c$ in $H_{t}$ with $P_{c} W(p)=W(q)$. Indeed, if $p=\exp t v_{x}, q=\exp t w_{y}$ with $v_{x}, w_{y} \in N_{1}(S)$, then there exists a piecewise geodesic $\alpha(s)$ on the soul with $\alpha(0)=x \quad \alpha(1)=y$ and a parallel vector field $v(s)$ along $\alpha(s)$ with $v(0)=v_{x}, v(1)=w_{y}$. Let $c:[0,1] \rightarrow H_{t}$ be defined by $c(s)=\exp t v(s)$. Since $t, s \rightarrow \exp t v(s)$ is totally geodesic, we see that $P_{c} W(p)=W(q)$. Since $c$ will in general not be contained in $U \cap H_{t}$ and since $H_{t}$ is not necessarily simply connected, it does not follow yet that 
$W$ is parallel, but we can conclude that $\left\langle W, W^{*}\right\rangle$ can take on only countably many values in $U \cap H_{t}$. Indeed $\left\langle P_{c} W(p), W^{*}\right\rangle$ only depends on the homotopy class of $\bar{c}$, where $\bar{c}$ is $c$ followed by a curve in $U \cap H_{t}$ from $c(1)$ to $p$, since $X$ is flat in a neighborhood of $H_{t}$. Thus $\left\langle W, W^{*}\right\rangle$ is constant on $U \cap H_{t}$ and $W=W^{*}$. Hence $H_{t}$ is totally geodesic and flat. The homotopy sequence for the $S^{1}$ bundle $S^{1} \rightarrow H_{t} \rightarrow S$ implies $\pi_{1}\left(H_{t}\right)=0$ or $\mathbf{Z}_{k}$ or $\mathbf{Z}$. Since $H_{t}$ is a compact flat manifold, it must be a circle. Thus $\operatorname{dim} X=2$ and $S$ is a point.

We now claim that all isometries of $X$ have a common fixed point $p_{0} \in X$. Indeed, $X$ contains a unique minimal, totally convex subset $C$ which contains the set where the curvature is positive. $C$ is invariant under all isometries of $X$ and hence the unique soul $p_{0}$ of $C$ in the sense of [CG] is fixed under all isometries. We construct a soul of $X$ in the sense of [CG] using all rays starting from $p_{0}$. Since the isometries of $X$ permute these rays, the soul of $X$ is a possibly different fixed point for the isometries of $X$, which we denote again by $p_{0}$.

Let now $M=\widetilde{M} / \Gamma$ where $\Gamma$ is the group of deck transformations. Every $\gamma \in \Gamma$ operates as $\left(\gamma_{1}, \gamma_{2}\right)$ on $\widetilde{M}=X \times \mathbf{R}^{n-2}$ and leaves the submanifold $\left\{p_{0}\right\} \times$ $\mathbf{R}^{n-2}$ invariant. Thus $\Gamma$ operates on $\mathbf{R}^{n-2}$ and this operation has compact quotient, since $M$ is flat outside a compact set. Thus $\Gamma$ is a Bieberbach group of rank $n-2$ and $\left\{p_{0}\right\} \times \mathbf{R}^{n-2} / \Gamma$ is a soul of $M$. Q.E.D.

Remark. Some of the above arguments in the $\operatorname{codim} S=2$ case can also be found in G. Walschap [W], where the $\operatorname{codim} S=2$ case in the soul theorem is considered in detail.

\section{REFERENCES}

[BGS] W. Ballmann, M. Gromov, and V. Schroeder, Manifolds of nonpositive curvature, Progress in Math., vol. 61, Birkhäuser, Boston, Mass., 1985.

[B] A. Besse, Einstein manifolds, Ergeb. Math. Grenzgeb., Band 10, Springer-Verlag, 1987.

[CE] J. Cheeger and D. G. Ebin, Comparison theorems in Riemannian geometry, North-Holland, Amsterdam, 1985.

[CG] J. Cheeger and D. Gromoll, On the structure of complete manifolds of nonnegative curvature, Ann. of Math. 96 (1972), 413-443.

[DZ] J. D'Atri and W. Ziller, Naturally reductive metrics and Einstein metrics on compact Lie groups, Mem. Amer. Math. Soc. No. 215 (1979).

[ES] J. H. Eschenburg and V. Schroeder, Riemannian manifolds with flat ends, Univ. of Münster, 1987, preprint.

[GW] R. E. Greene and H. Wu, Gap theorems for noncompact Riemannian manifolds, Duke Math. J. 49 (1982), 731-756.

[GWZ] H. Gluck, F. Warner, and W. Ziller, The geometry of the Hopf fibrations, L'Enseignement Math. 32 (1986), 173-198.

[K] B. Kostant, On convexity, the Weyl group, and the Iwasawa decomposition, Ann. Sci. École Norm. Sup. 6 (1973), 413-455. 
[T] V. A. Toponogov, Computation of the length of a closed geodesic on a convex surface, Dokl. Akad. Nauk SSSR 124 (1959), 282-284.

[W] G. Walschap, Nonnegatively curved manifolds with souls of codimension 2 , Stony Brook, 1986, preprint.

Mathematisches Institut, Universität Münster, Einsteinstr. 62, D-4400 Münster, FEDERAL RePUblic OF Germany

Department of Mathematics, University of Pennsylvania, Philadelphia, PennsylvaNIA 19104-6395 